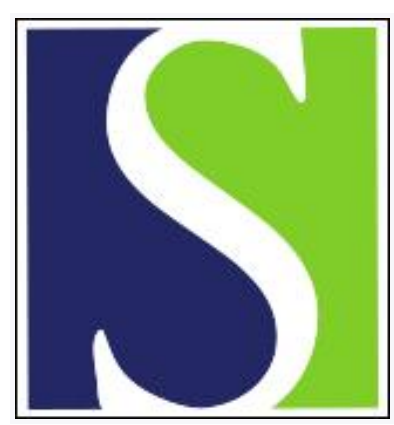

Scand J Work Environ Health 2013;39(1):76-87

https://doi.org/10.5271/sjweh.3291

Published online: 15 Mar 2012, Issue date: Jan 2013

Predictors of employment among cancer survivors after medical rehabilitation - a prospective study

by Mehnert A, Koch U

Affiliation: Department and Outpatient Clinic of Medical Psychology, University Medical Center Hamburg-Eppendorf, Martinistrasse 52, 20246 Hamburg, Germany. mehnert@uke.de

The following article refers to this text: 2020;46(4):382-391

Key terms: cancer; cancer survivor; cancer survivorship; employment; predictor; prospective study; psycho-oncology; rehabilitation; return to work; RTW

This article in PubMed: www.ncbi.nlm.nih.gov/pubmed/22422040

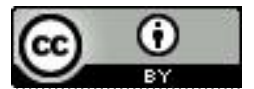




\title{
Predictors of employment among cancer survivors after medical rehabilitation - a prospective study
}

\author{
by Anja Mehnert, PhD, ${ }^{1}$ Uwe Koch, PhD, MD 1,2
}

\begin{abstract}
Mehnert A, Koch U. Predictors of employment among cancer survivors after medical rehabilitation - a prospective study. Scand J Work Environ Health. 2013;39(1):76-87. doi:10.5271/sjweh.3291

Objectives This study aimed to (i) investigate cancer survivor's employment status one year after the completion of a medical rehabilitation program and (ii) identify demographic, cancer, and psychosocial, treatment-, and work-related predictors of return to work (RTW) and time until RTW.
\end{abstract}

Methods A total of 1520 eligible patients were consecutively recruited on average 11 months post diagnosis and assessed at the beginning $\left(\mathrm{t}_{0}\right)(\mathrm{N}=1148)$ and end of rehabilitation $\left(\mathrm{t}_{1}\right)(\mathrm{N}=1060)$ and 12 months after rehabilitation $\left(\mathrm{t}_{2}\right)(\mathrm{N}=750)$. Participants completed validated measures assessing functional impairments, pain, anxiety, depression, quality of life, social support, and work-related characteristics including work ability, sick leave absence, job requirements, work satisfaction, self-perceived employer accommodation, and perceived job loss. Physicians estimated the degree of cancer-entity-specific functional impairment.

Results In a mean time of six weeks after rehabilitation, 568 patients $(76 \%)$ had returned to work. The multivariate hierarchical logistic regression analysis indicated that baseline RTW intention [odds ratio (OR) $6.22,95 \%$ confidence interval ( $95 \% \mathrm{CI}$ ) 1.98-19.51], perceived employer accommodation (OR 1.93, 95\% CI 0.33-0.99), high job requirements ( $\mathrm{OR}=1.84,95 \% \mathrm{CI} 1.02-3.30)$, cancer recurrence or progression $(\mathrm{OR}=0.27,95 \% \mathrm{CI} 0.12$ $0.63)$, baseline sick leave absence $(\mathrm{OR}=0.26,95 \% \mathrm{CI} 0.09-0.77)$, and problematic social interactions $(\mathrm{OR}=0.58$, $95 \%$ CI $0.33-0.99$ ) emerged as significant predictors for RTW. The explained variance of the total model was Nagelkerke's $\mathrm{R}^{2}=0.59(\mathrm{P}<0.001)$.

Conclusion Our findings emphasize the high relevance of motivational factors. Occupational motivation and skepticism towards returning to work should be carefully assessed at the planning of the rehabilitation program.

Key terms cancer survivorship; psycho-oncology; return to work; RTW.

Since an increasing number of patients are likely to return to work after diagnosis and treatment completion, there is an increasing recognition of the short and long-term impact of cancer and both its physical and psychosocial consequences on employment during the last years (1-13). Unfavorable cancer and treatment consequences include a variety of physical and functional disabilities, and psychological distress that may adversely affect a patient's work ability, work satisfaction, as well as employment status (14-17). Since work has the potential to help patients regain a sense of meaning, normalcy and being valued, returning to work may comprise a range of positive consequences for the recovery and the psychological well-being $(18,19)$.
Previous research suggested that cancer increases the risk of unemployment among survivors compared to healthy controls (20-22). On average, $63.5 \%$ of cancer survivors (range 24-94\%) return to work (17). Overall, studies indicate a steady increase of return to work (RTW) from on average $40 \%$ at six months post diagnosis to $62 \%$ at 12 months, $73 \%$ at 18 months, and $89 \%$ at 24 months. Factors significantly associated with a greater likelihood of being employed or RTW include perceived employer accommodation, flexible working arrangements, counseling, training and rehabilitation services, younger age, higher education, male gender, a lower physical symptom burden, cancer remission, shorter length of sick leave, and continuity of care $(7,14,17,23-28)$.

1 Department and Outpatient Clinic of Medical Psychology, Center for Psychosocial Medicine and University Cancer Center Hamburg (UCCH), University Medical Center Hamburg-Eppendorf, Hamburg, Germany.

2 Deanery Medical Faculty and Department and Outpatient Clinic of Medical Psychology, Center for Psychosocial Medicine, University Medical Center Hamburg-Eppendorf, Hamburg, Germany.

Correspondence to: Anja Mehnert PhD, Department and Outpatient Clinic of Medical Psychology, University Medical Center HamburgEppendorf, Martinistrasse 52, 20246 Hamburg, Germany. [E-mail: mehnert@uke.de] 
However, although important findings have emerged in previous studies on work and employment among cancer survivors $(7,29,30)$, only limited knowledge exists about RTW after rehabilitation taking into account the impact of demographic characteristics, cancer and treatment-related physical factors, psychosocial as well as of work-related aspects of employment and RTW. The Word Health Organization (WHO) (31) has defined rehabilitation as "the use of all means aimed at reducing the impact of disabling and handicapping conditions and at enabling people with disabilities to achieve optimal social integration". Medical rehabilitation programs in Germany are provided to cancer patients according to the overall aim defined by the WHO and the International Classification of Functioning, Disability and Health (ICF) (31). Thus, the (re-)integration of individuals with disabilities, chronic health conditions, diseases, and handicaps into society and working life is one important aspect of rehabilitation by eliminating or reducing the impact of chronic illness and disability. The aim is to maintain a patient's optimal physical, sensory, psychological, and social functional levels. Rehabilitation also serves to prevent an impending disability or the aggravation of existing physical damages.

Based on social laws in Germany, cancer patients have a legal right to participate in at least one rehabilitation program after the completion of primary cancer treatments (32). Traditionally, cancer rehabilitation programs are mainly carried out in in-patient settings in specialized rehabilitation clinics. Access to cancer rehabilitation programs is usually facilitated by hospital doctors and social workers immediately after completion of the primary treatment ("follow-up rehabilitation"). However, cancer rehabilitation at a later stage during the course of cancer treatment is also provided. Rehabilitation costs are covered mainly by the pension and health insurances. A cancer rehabilitation program lasts three weeks following a multidimensional therapeutic approach that includes patient education, exercises, and physical therapy to regain physical fitness and vitality, relaxation training, psychosocial counseling, and psychosocial support groups to enhance coping skills as well as individual psychotherapy.

This prospective longitudinal study aimed to (i) identify the employment rate 12 -months after cancer rehabilitation, (ii) explore the work situation and experienced work changes, and (iii) identify demographic, medical, functional, psychosocial, and work-related predictors of the likelihood and time period of RTW.

\section{Methods}

The study received research ethics committee approval. All patients provided written informed consent prior to participation. Consecutive patients were recruited from four cancer rehabilitation facilities and assessed at the beginning $\left(t_{0}\right)$ and end of rehabilitation $\left(t_{1}\right)$, and 12 months after rehabilitation $\left(\mathrm{t}_{2}\right)$. Inclusion criteria comprised (i) age 18-60 years, (ii) the capability to complete study measures, and (iii) the absence of permanent invalidity and early retirement. Since the old age pension in Germany generally becomes effective at 65 years, the age limit of 60 years was chosen to enable patients to have a sufficient time period left to return to work.

A total of 1148 patients were enrolled at $t_{0}(75.5 \%$ participation rate), whereas 372 patients declined to participate. Among those who participated at $t_{0}, 1060$ completed the questionnaires at $\mathrm{t}_{1}$. At $\mathrm{t}_{2}$, questionnaires were mailed to an eligible 994 patients (36 patients had moved to an unknown address and 30 had died), $750(75 \%, 65 \%$ of the total sample) of whom returned questionnaires that could be evaluated (figure 1). Table 1 presents baseline sample characteristics.

\section{Non-responder analyses}

At $\mathrm{t}_{0}$, participants and non-participants differed in terms of age [mean 48.5, standard deviation (SD) 7.2, years versus mean 50.4, SD 6.1, years] $(\mathrm{P}<0.001)(\mathrm{d}=0.3)$ and male gender $(16 \%$ versus $22 \%)(\mathrm{P}=0.007)(\varphi=0.07)$. No group differences in cancer entities were observed. At $\mathrm{t}_{2}$ non-participating patients were more likely to be male $(\mathrm{P}=0.008)(\varphi=0.09)$, widowed $(\mathrm{P}=0.03)(\varphi=0.10)$, and have head and neck or lung cancer $(\mathrm{P}=0.005)(\varphi=0.14)$. Participants at $t_{2}$ were found to be significantly less depressed $(\mathrm{P}=0.01)\left(\eta^{2}=0.007\right)$ and had a lower level of fear of cancer recurrence $(\mathrm{P}=0.009)\left(\eta^{2}=0.007\right)$.

\section{Study variables and measures}

The main outcomes for this study were employment at 12 months after rehabilitation $(\mathrm{M}=23.1$ months after cancer diagnosis) and time until RTW. Employment was defined according to a patient's positive response to the question “Are you currently working?" Time until RTW was measured in weeks after rehabilitation.

Demographic information was obtained at baseline consisting of standardized questions concerning (age, gender, marital status, and employment history). Education, monthly household net income, and occupational position were used to calculate a 3 -factor social status index (33). Medical information was collected at baseline [cancer entity, months since diagnosis, cancer stage as defined by the International Union against Cancer (UICC), clinical characteristics and disease phase]. In addition to the baseline Karnofsky performance status (34), physicians estimated the degree of functional impairment using cancer-entity-specific physical functioning scales. The Karnofsky status is a performance 


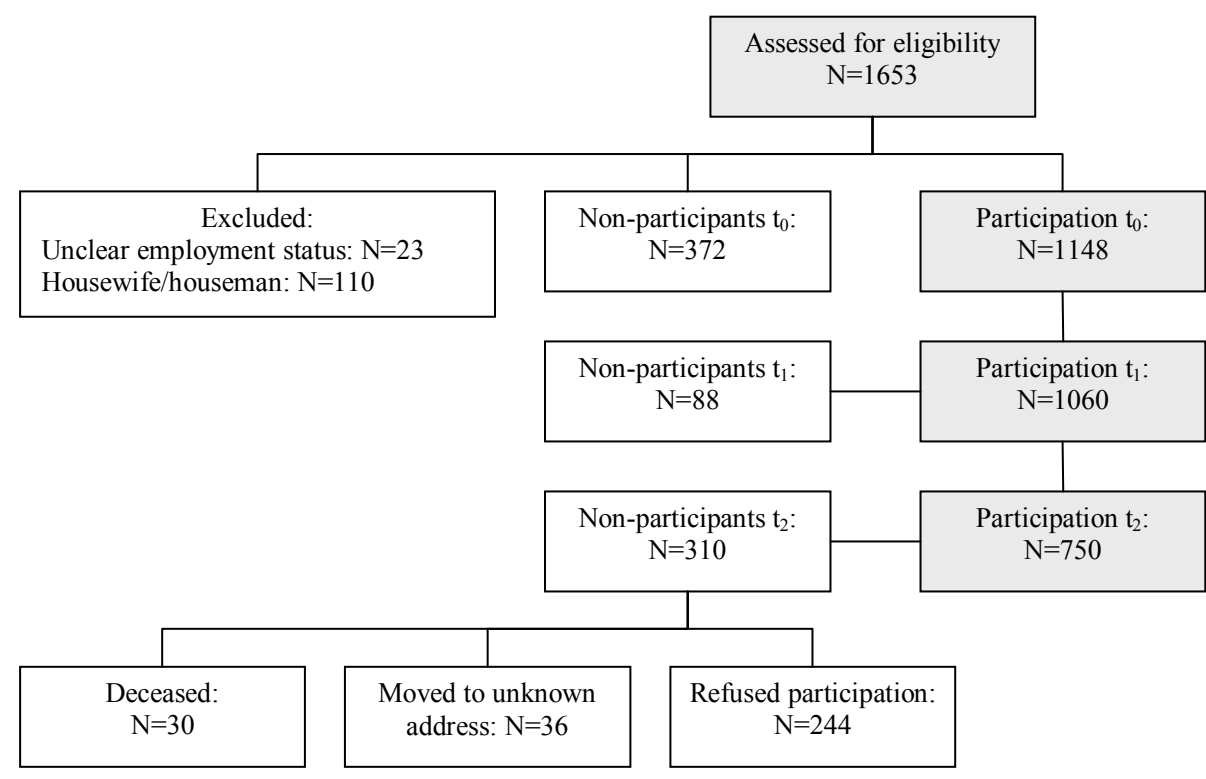

Figure 1. Enrollment of cancer survivors $\left(t_{0}=\right.$ beginning of cancer rehabilitation, $t_{1}=$ end of a 3-4 week cancer rehabilitation, $\mathrm{t}_{2}=12$ months after cancer rehabilitation) measure for rating the ability of a somatically ill person to perform usual activities. A person is evaluated on a score of $0-100$, where $0=$ dead and $100=$ normal, no complaints, no signs of disease. Using cancer-entityspecific physical functioning scales, the physician measures the limitations specific for each tumor entity such as shoulder mobility or lymphedema in breast cancer, incontinence in genital or colon cancers, swallowing problems in head and neck cancers or dyspnea in lung cancer. Pain intensity during the last week was evaluated using the Brief Pain Inventory (BPI) (35).

The psychosocial and work-related variables were further assessed at baseline. Anxiety and depression were assessed using the Hospital Anxiety and Depression Scale (HADS) (36). Fear of cancer recurrence was measured using the 12-item short version of the Fear of Recurrence Questionnaire (FoP-Q-SF) scored on a 5 -point Likert scale ranging from $1=$ never to $5=$ very often (37). The Short-Form Health Survey assesses dimensions of quality of life (QoL): Here, the two summary scores for physical (PCS) and mental health (MCS) were calculated. Higher scores indicate better QoL (38). The Illness-Specific Social Support Scale (ISSS) measures the degree to which partners/friends provide positive support (eg, "listened to you") or act in a non-supportive way (detrimental interactions) (eg, "tried to change the way you're coping with your illness in a way you didn't like"). Items are scored on a 5-point Likert scale ranging from $0=$ ever to $4=$ always (39).

Occupational and work-related characteristics were assessed using questions and brief questionnaires developed and psychometrically evaluated by Bürger et al (40). Occupational information included (i) professional status, (ii) work ability and periods of sick leave absence, (iii) 10 items about job requirements (eg, many work responsibilities, high pressure of competition, tight schedules) answered on a 4-point Likert scale ranging from $1=$ =almost never to $4=$ quite often (Cronbach's $\alpha=0.87$ ), and (iv) 12 items about work satisfaction answered on a 7-point Likert scale ranging from $1=$ not satisfied at all to $7=$ totally satisfied (Cronbach's $\alpha=0.92)$. Self-perceived work ability was evaluated on a 5-point Likert item ranging from $1=$ totally limited to $5=$ not limited at all; self-perceived employer accommodation was evaluated on a 5-point Likert item ranging from $1=$ not at all to $5=$ extremely. Perceived threat of job loss was measured using single-item questions (No/Yes).

\section{Statistical analysis}

In order to identify significant predictors of RTW, demographic, medical, functional, psychosocial, and workrelated factors were entered separately (block-wise) into a multivariate hierarchical logistic regression analysis against the outcome variable "RTW". Step-wise backwards elimination was used, testing each candidate variable for removal using Wald statistic. Before testing the regression model, we performed correlations (Pearson and Spearmans correlation coefficients) for all predictor candidates between variables in one block and between candidate variables and the outcome criteria. In order to identify significant predictors of the time period of RTW, candidate predictor variables were entered into a Cox's proportional hazards model to calculate hazard ratios. To provide an estimate of the magnitude of the group differences, Cohen's standardized effect size $(\varphi$, $\left.\mathrm{d}, \eta^{2}\right)$ was calculated. Two-tailed significance tests were conducted using a significance level of $\mathrm{P}<0.05$. 
Table 1. Baseline demographic and medical sample characteristics. [SD=standard deviation; UICC=International Union against Cancer]

\begin{tabular}{|c|c|c|c|c|c|c|c|c|c|c|c|c|c|c|}
\hline & \multicolumn{12}{|c|}{ Study sample } & \multirow{3}{*}{$\begin{array}{c}\mathrm{P}- \\
\text { value }\end{array}$} & \multirow[t]{3}{*}{$d / \varphi$} \\
\hline & \multicolumn{4}{|c|}{ Total sample $(\mathrm{N}=750)$} & \multicolumn{4}{|c|}{ Employed (N=702) } & \multicolumn{4}{|c|}{ Unemployed $(\mathrm{N}=48)$} & & \\
\hline & Mean & SD & $\mathrm{N}$ & $\%$ & Mean & SD & $\mathrm{N}$ & $\%$ & Mean & SD & $\mathrm{N}$ & $\%$ & & \\
\hline \multicolumn{15}{|l|}{ Demographic characteristics } \\
\hline Age & 48.7 & 6.8 & & & 48.7 & 6.7 & & & 48.8 & 8.0 & & & 0.93 & \\
\hline Female gender & & & 643 & 85.7 & & & 597 & 85.0 & & & 46 & 95.8 & 0.04 & 0.08 \\
\hline Married & & & 498 & 66.4 & & & 473 & 67.4 & & & 25 & 52.0 & 0.03 & 0.11 \\
\hline Partnership & & & 566 & 75.5 & & & 537 & 76.5 & & & 29 & 60.4 & 0.01 & 0.09 \\
\hline High school/university degree & & & 218 & 29.1 & & & 207 & 29.5 & & & 11 & 22.9 & 0.55 & \\
\hline Monthly household net income $(€)$ & & & & & & & & & & & & & $<0.001$ & 0.44 \\
\hline$<1000$ & & & 39 & 9.3 & & & 18 & 4.9 & & & 21 & 43.8 & & \\
\hline $1000-<2000$ & & & 136 & 32.5 & & & 122 & 33.0 & & & 14 & 29.2 & & \\
\hline $2000-<3000$ & & & 124 & 29.7 & & & 115 & 31.1 & & & 9 & 18.8 & & \\
\hline$\geq 3000$ & & & 119 & 28.5 & & & 115 & 31.1 & & & 4 & 8.3 & & \\
\hline \multicolumn{15}{|l|}{ Social class } \\
\hline Lower & & & 203 & 27.1 & & & 176 & 25.1 & & & 27 & 56.3 & $<0.001$ & 0.18 \\
\hline Middle & & & 441 & 58.8 & & & 421 & 60.0 & & & 20 & 41.7 & & \\
\hline Upper & & & 106 & 14.1 & & & 105 & 15.0 & & & 1 & 2.1 & & \\
\hline \multicolumn{15}{|l|}{ Medical characteristics } \\
\hline Cancer entity & & & & & & & & & & & & & & \\
\hline Breast cancer & & & 446 & 59.5 & & & 419 & 59.7 & & & 27 & 56.3 & 0.53 & \\
\hline Gynecological cancers & & & 109 & 14.5 & & & 100 & 14.2 & & & 9 & 18.8 & & \\
\hline Head and neck cancers & & & 67 & 8.9 & & & 62 & 8.8 & & & 5 & 10.4 & & \\
\hline Skin cancer & & & 46 & 6.1 & & & 45 & 6.4 & & & 1 & 2.1 & & \\
\hline Colon/rectum cancer & & & 42 & 5.6 & & & 39 & 5.6 & & & 3 & 6.3 & & \\
\hline Lung cancer & & & 23 & 3.1 & & & 20 & 2.8 & & & 3 & 6.3 & & \\
\hline Hematological neoplasias & & & 17 & 2.3 & & & 17 & 2.4 & & & 0 & & & \\
\hline Months since diagnosis & 11.1 & 8.5 & & & 11.1 & 8.5 & & & 10.2 & 7.3 & & & 0.47 & \\
\hline Cancer stage $\left(\right.$ UICC) ${ }^{\mathrm{a}}$ & & & & & & & & & & & & & 0.20 & \\
\hline In situ & & & 8 & 1.1 & & & 6 & 0.9 & & & 2 & 4.2 & & \\
\hline I & & & 305 & 41.6 & & & 288 & 42.0 & & & 17 & 35.4 & & \\
\hline$\|$ & & & 268 & 36.6 & & & 248 & 36.2 & & & 20 & 41.7 & & \\
\hline III & & & 118 & 16.1 & & & 112 & 16.4 & & & 6 & 12.5 & & \\
\hline IV & & & 34 & 4.6 & & & 31 & 4.5 & & & 3 & 6.3 & & \\
\hline \multicolumn{15}{|l|}{ Clinical characteristics } \\
\hline First primary tumor & & & 719 & 95.9 & & & 673 & 95.9 & & & 46 & 95.8 & 0.99 & \\
\hline Second primary tumor & & & 31 & 4.1 & & & 29 & 4.1 & & & 2 & 4.2 & & \\
\hline Disease phase & & & & & & & & & & & & & 0.61 & \\
\hline Remission & & & 653 & 87.1 & & & 612 & 87.2 & & & 41 & 85.4 & & \\
\hline Recurrence/progress/metastasis & & & 72 & 9.6 & & & 68 & 9.7 & & & 1 & 2.1 & & \\
\hline Unclear & & & 25 & 3.3 & & & 22 & 3.1 & & & 3 & 6.3 & & \\
\hline Treatment phase & & & & & & & & & & & & & 0.57 & \\
\hline Curative treatment intention & & & 693 & 92.4 & & & 648 & 92.3 & & & 45 & 93.8 & & \\
\hline Palliative treatment intention & & & 36 & 4.8 & & & 35 & 5.0 & & & 1 & 2.1 & & \\
\hline Unclear & & & 21 & 2.8 & & & 19 & 2.7 & & & 2 & 4.2 & & \\
\hline $\begin{array}{l}\text { Months since active cancer } \\
\text { treatment }\end{array}$ & 7.7 & 12.5 & & & 7.7 & 12.6 & & & 6.5 & 10.4 & & & 0.52 & \\
\hline \multicolumn{15}{|l|}{ Cancer treatments } \\
\hline Surgery & & & 716 & 95.5 & & & 669 & 95.3 & & & 47 & 97.9 & 0.40 & \\
\hline Radiation therapy & & & 494 & 65.9 & & & 464 & 66.1 & & & 30 & 62.5 & 0.61 & \\
\hline Chemotherapy & & & 454 & 60.5 & & & 427 & 60.8 & & & 27 & 56.3 & 0.53 & \\
\hline Hormonal treatment & & & 328 & 43.7 & & & 308 & 43.9 & & & 20 & 41.7 & 0.77 & \\
\hline Total number of therapies & 2.7 & 1.1 & & & 2.7 & 1.1 & & & 2.6 & 1.1 & & & 0.58 & \\
\hline Karnofsky performance status & 91.7 & 7.5 & & & 91.7 & 7.5 & & & 91.5 & 6.2 & & & 0.81 & \\
\hline
\end{tabular}

a Without hematological neoplasias. 


\section{Results}

\section{Baseline occupational characteristics}

At the beginning of the rehabilitation program, 702 patients (93.6\%) were employed and 48 (6.4\%) were unemployed. Among the employed, 54.9\% were on physician-classified sick leave (usually the primary care physician). The majority of the working participants worked as employees (75.1\%), 18.4\% were workers; $5.6 \%$ were self-employed, and $1.0 \%$ worked as civil servants. The mean duration of sick leave within the 12-months period prior to the rehabilitation program was 150.6 (SD 107.4, range 1-365) days. The majority of patients $(84.5 \%)$ were motivated either to return to work or be re-employed after rehabilitation.

\section{RTW and re-employment 12 months after rehabilitation}

At $t_{2}, 568$ patients $(75.7 \%)$ had returned to work or were re-employed. The percentage of patients who returned to work was highest among participants not on sick leave at baseline (92.4\%). Twenty-one percent of the patients who were unemployed at baseline managed to get employed at a follow-up time point (table 4). Patients who returned to work or were re-employed were slightly younger [mean 48.2, (SD 7.0) years versus mean 50.5 $(\mathrm{SD} 6.1)$ years] $(\mathrm{P}<0.001)\left(\eta^{2}=0.021\right)$. No gender differences were observed. The highest percentage of patients who returned to work was observed among employ- ees $(97.2 \%)$, and the lowest percentage among workers $(66.4 \%)(\mathrm{P}=0.02)(\varphi=0.12)$. No group differences were found in clinical characteristics. However, among patients with a higher UICC cancer stage $(\mathrm{P}<0.001)$ $(\varphi=0.18)$ and palliative treatment, a lower percentage $(38.9 \%)$ returned to work or were re-employed compared to patients with curative treatment (78.1\%) $(\mathrm{P}<0.001)(\varphi=0.20)$. Also, the highest percentage of patients who did not return to work was observed among patients with lung cancer (43\%) and head and neck cancers $(58 \%)$. Among patients with cancer progress or metastatic cancer, a significantly lower percentage $(38.9 \%)$ returned to work or got re-employed compared to patients in remission $(78.6 \%)(\mathrm{P}<0.001)(\varphi=0.17)$.

\section{RTW time period and re-employment after rehabilitation}

Forty-nine percent of patients returned to work immediately after rehabilitation. The mean time until RTW or re-employment was 5.7 (SD 8.6, range 1-45) weeks. The highest percentage of patients who returned to work immediately were among those who were not on sick leave at baseline, belonged to the upper social class, and had skin cancer (table 2).

\section{Work situation at follow-up}

Among the 568 patients who returned to work, the majority $(81.2 \%)$ returned to their former position and workplace; $46 \%$ worked full-time. More women (77.8\%)

Table 2. Time period of return to work (RTW) and reemployment after rehabilitation ( $N=568)$.

\begin{tabular}{|c|c|c|c|c|c|c|c|}
\hline \multirow[t]{3}{*}{ Employed patients } & \multirow[t]{3}{*}{$\mathrm{N}\left(\mathrm{t}_{0}\right)$} & \multicolumn{4}{|c|}{ Time period to RTW / re-employment } & \multirow[t]{3}{*}{ P-value } & \multirow[t]{3}{*}{$\varphi / \eta^{2}$} \\
\hline & & \multicolumn{2}{|c|}{$\begin{array}{l}\text { Patients who returned to work } \\
\text { immediately after rehabilitation }\end{array}$} & \multicolumn{2}{|c|}{$\begin{array}{l}\text { Weeks until RTW / } \\
\text { re-employment }\end{array}$} & & \\
\hline & & $\mathrm{N}$ & $\%$ & Mean & SD & & \\
\hline Baseline employment status & & & & & & $<0.001$ a $/<0.001$ b & $0.68^{a} / 0.24 b$ \\
\hline Patients working & 268 & 228 & 85.1 & 1.64 & 2.46 & & \\
\hline Patients on sick leave & 290 & 48 & 16.6 & 8.90 & 9.83 & & \\
\hline Unemployed patients & 10 & 2 & 20.0 & 21.60 & 16.67 & & \\
\hline Total & 568 & 278 & 48.9 & 5.68 & 8.60 & & \\
\hline Social class & & & & & & $0.008^{a} / 0.63^{b}$ & $0.13^{a}$ \\
\hline Lower social class & 133 & 53 & 39.8 & 6.23 & 8.23 & & \\
\hline Middle social class & 343 & 169 & 49.3 & 5.41 & 8.44 & & \\
\hline Upper social class & 92 & 56 & 60.9 & 5.89 & 9.64 & & \\
\hline Cancer entity & & & & & & $0.001^{a} /<0.001^{b}$ & $0.20^{a} / 0.05^{b}$ \\
\hline Breast cancer & 348 & 171 & 49.1 & 5.19 & 7.98 & & \\
\hline Gynecological cancers & 81 & 38 & 46.9 & 5.23 & 6.77 & & \\
\hline Head and neck cancers & 39 & 14 & 35.9 & 8.03 & 10.31 & & \\
\hline Skin cancer & 38 & 30 & 78.9 & 2.76 & 6.00 & & \\
\hline Colon/rectum cancer & 36 & 16 & 44.4 & 10.21 & 13.41 & & \\
\hline Lung cancer & 10 & 1 & 10.0 & 13.70 & 14.21 & & \\
\hline Hematological neoplasias & 16 & 8 & 50.0 & 5.13 & 7.53 & & \\
\hline
\end{tabular}

a Significance and effect size refer to group differences in patients who returned to work immediately after rehabilitation.

b Significance and effect size refer to group differences in weeks until RTW/reemployment. 
worked part-time compared to $22.2 \%$ of men $(\mathrm{P}<0.001)$ $(\varphi=0.25)$. Fifty-two percent of the patients were on sick leave absence at least once after rehabilitation for an average duration of 62 days.

\section{Predictors of RTW - logistic regression model}

Before testing the logistic regression model, we performed correlations between variables in one block for all predictor candidates. In candidate predictor variables with an intercorrelation of $r \geq 0.60$, one variable was removed from the regression analysis in order to avoid multicollinearity. The following variables were removed: marital status, treatment phase (curative ver- sus palliative treatment intention), months since active cancer treatment, total numbers of therapy, anxiety, distress, and the expected period of RTW. Table 3 presents the correlations between the final candidate predictor variables and the outcome variables.

The first block entered into the multivariate hierarchical logistic regression model consisted of demographic factors (table 4). The variables education and social status were excluded from the regression model, whereas age and monthly household net income remained in the model (Nagelkerke's $\left.\mathrm{R}^{2}=0.07\right)(\mathrm{P}<0.001)$.

The second block entered consisted of medical and functional factors. The variables cancer entity, months since current diagnosis, UICC cancer stage, and number

Table 3. Correlations between candidate predictor variables $\left(\mathrm{t}_{0}\right)$ and outcome variables. [UICC=International Union against Cancer.]

\begin{tabular}{|c|c|c|c|c|}
\hline \multirow[t]{2}{*}{ Candidate predictor variables $\left(\mathrm{t}_{0}\right)$} & \multicolumn{2}{|c|}{ 12-months return to work } & \multicolumn{2}{|c|}{ Time period of return to work (weeks) } \\
\hline & $r$ & P-value & $r$ & P-value \\
\hline \multicolumn{5}{|l|}{ Demographic factors } \\
\hline Age & -0.14 & $>0.001$ & -0.13 & $<0.001$ \\
\hline Education & 0.12 & 0.001 & -0.12 & 0.001 \\
\hline Monthly household net income & 0.18 & $>0.001$ & -0.20 & $<0.001$ \\
\hline Social status & 0.16 & $>0.001$ & -0.16 & $<0.001$ \\
\hline \multicolumn{5}{|l|}{ Medical and functional factors } \\
\hline \multicolumn{5}{|l|}{ Cancer entity } \\
\hline Breast cancer & 0.07 & 0.08 & -0.08 & 0.06 \\
\hline Gynecological cancers & -0.01 & 0.71 & 0.03 & 0.55 \\
\hline Head and neck cancers & -0.13 & $>0.001$ & 0.05 & 0.26 \\
\hline Skin cancer & 0.04 & 0.26 & -0.09 & 0.05 \\
\hline Colon/rectum cancer & 0.06 & 0.12 & 0.11 & 0.01 \\
\hline Lung cancer & -0.13 & $>0.001$ & 0.13 & 0.003 \\
\hline Hematological neoplasias & 0.07 & 0.07 & -0.03 & 0.52 \\
\hline Months since current diagnosis & 0.09 & 0.03 & -0.30 & $>0.001$ \\
\hline Cancer stage (UICC) & -0.15 & $>0.001$ & -0.15 & $>0.001$ \\
\hline \multicolumn{5}{|l|}{ Disease phase } \\
\hline Remission & 0.17 & $>0.001$ & 0.007 & 0.87 \\
\hline Recurrence/progress/metastasis & -0.12 & 0.004 & 0.07 & 0.12 \\
\hline Number of functional impairments & -0.19 & $>0.001$ & 0.26 & $>0.001$ \\
\hline Number of physical problems & -0.19 & $>0.001$ & 0.26 & $>0.001$ \\
\hline Karnofsky status & 0.30 & $>0.001$ & -0.34 & $>0.001$ \\
\hline Pain & -0.25 & $>0.001$ & 0.32 & $>0.001$ \\
\hline \multicolumn{5}{|l|}{ Psychosocial factors } \\
\hline Fear of cancer recurrence & -0.21 & $>0.001$ & 0.08 & 0.05 \\
\hline Depression & -0.31 & $>0.001$ & 0.09 & 0.03 \\
\hline Mental quality of life & 0.23 & $>0.001$ & -0.06 & 0.17 \\
\hline Physical quality of life & 0.32 & $>0.001$ & -0.24 & $>0.001$ \\
\hline Social support & 0.10 & 0.007 & 0.02 & 0.71 \\
\hline Detrimental interactions & -0.21 & $>0.001$ & 0.04 & 0.32 \\
\hline \multicolumn{5}{|l|}{ Work-related factors } \\
\hline \multicolumn{5}{|l|}{ Employment and work ability } \\
\hline Working & 0.31 & $>0.001$ & -0.63 & $>0.001$ \\
\hline Sick leave & -0.14 & $>0.001$ & 0.46 & $>0.001$ \\
\hline Unemployed & -0.34 & $>0.001$ & 0.30 & $>0.001$ \\
\hline Duration of sick leave (days) prior to rehabilitation & -0.30 & $>0.001$ & 0.47 & $>0.001$ \\
\hline Self-perceived work ability & -0.48 & $>0.001$ & 0.37 & $>0.001$ \\
\hline Perceived threat of job loss & -0.17 & $>0.001$ & 0.02 & 0.64 \\
\hline Intention to return to work & 0.50 & $>0.001$ & -0.05 & 0.28 \\
\hline Expected period of return to work after rehabilitation & -0.33 & $>0.001$ & 0.53 & $>0.001$ \\
\hline Perceived employer accommodation & 0.34 & $>0.001$ & -0.07 & 0.13 \\
\hline Job requirements & -0.11 & 0.004 & 0.08 & 0.06 \\
\hline Overall job satisfaction & 0.13 & $>0.001$ & -0.02 & 0.65 \\
\hline
\end{tabular}


Table 4. Multivariate logistic regression model for the identification of significant predictors of 12-months (re-) employment after rehabilitation. [OR=0dds ratio; $\mathrm{SD}=$ standard deviation; $\mathrm{SE}=$ standard error; $95 \% \mathrm{Cl}=95 \%$ confidence interval]

\begin{tabular}{|c|c|c|c|c|c|c|c|c|c|c|c|c|c|}
\hline & \multicolumn{8}{|c|}{ (Re-)employment } & \multirow[t]{3}{*}{ B } & \multirow[t]{3}{*}{ SE } & \multirow{3}{*}{ OR } & \multirow{3}{*}{$95 \% \mathrm{Cl}$} & \multirow[t]{3}{*}{ P-value } \\
\hline & \multicolumn{4}{|c|}{ Yes $(\mathrm{N}=568)$} & \multicolumn{4}{|c|}{ No $(\mathrm{N}=182)$} & & & & & \\
\hline & Mean & $\mathrm{SD}$ & $\mathrm{N}$ & $\%$ & Mean & SD & $\mathrm{N}$ & $\%$ & & & & & \\
\hline $\begin{array}{l}\text { Block } 1 \text { Demographic } \\
\text { factors (Nagelkerke's } \\
\mathrm{R}^{2}=0.07 \text { ) }\end{array}$ & & & & & & & & & & & & & $<0.001$ \\
\hline $\begin{array}{l}\text { Age } \\
\text { Monthly household net } \\
\text { income }(€)\end{array}$ & 48.2 & 7.0 & & & 50.5 & 6.1 & & & -0.05 & 0.03 & 0.94 & $0.90-1.00$ & 0.054 \\
\hline$<1000^{b}$ & & & 29 & 46.8 & & & 33 & 53.2 & & & & & 0.21 \\
\hline $1000-<2000$ & & & 183 & 73.8 & & & 65 & 26.2 & 1.20 & 0.72 & 3.33 & $0.87-13.73$ & 0.10 \\
\hline $2000-<3000$ & & & 190 & 78.5 & & & 52 & 21.5 & 0.74 & 0.70 & 2.09 & $0.53-8.31$ & 0.29 \\
\hline$\geq 3000$ & & & 166 & 83.8 & & & 32 & 16.2 & 0.41 & 0.72 & 1.50 & $0.37-6.18$ & 0.57 \\
\hline $\begin{array}{l}\text { Block } 2 \text { Medical and } \\
\text { functional factors } \\
\text { (Nagelkerke's } R^{2}=0.32 \text { ) }\end{array}$ & & & & & & & & & & & & & $<0.001$ \\
\hline \multicolumn{14}{|l|}{ Disease phase } \\
\hline $\begin{array}{l}\text { Remissiona } \\
\text { Recurrence/progress/ } \\
\text { metastasis }\end{array}$ & & & $\begin{array}{r}513 \\
55\end{array}$ & $\begin{array}{l}10.0 \\
56.7\end{array}$ & & & $\begin{array}{r}140 \\
42\end{array}$ & $\begin{array}{l}21.4 \\
43.3\end{array}$ & -1.31 & 0.43 & 0.27 & $0.12-0.63$ & \\
\hline $\begin{array}{l}\text { Number of functional } \\
\text { impairments }\end{array}$ & & & 2.3 & 3.0 & & & 4.2 & 4.6 & -0.01 & 0.04 & 1.00 & $0.91-1.08$ & 0.78 \\
\hline Karnofsky status & 93.0 & 7.5 & & & 87.8 & 7.1 & & & 0.05 & 0.02 & 1.05 & $1.01-1.09$ & 0.01 \\
\hline Pain & 2.5 & 2.1 & & & 4.1 & 2.8 & & & -0.09 & 0.09 & 0.91 & $0.77-1.08$ & 0.28 \\
\hline Physical quality of life & 50.6 & 9.0 & & & 43.7 & 7.8 & & & 0.02 & 0.03 & 1.02 & $0.99-1.08$ & 0.51 \\
\hline $\begin{array}{l}\text { Block } 3 \text { Psychosocial } \\
\text { factors (Nagelkerke's } \\
\mathrm{R}^{2}=0.39 \text { ) }\end{array}$ & & & & & & & & & & & & & $<0.001$ \\
\hline Mental quality of life & 53.2 & 10.1 & & & 47.6 & 11.1 & & & 0.03 & 0.02 & 1.03 & $1.00-1.07$ & 0.08 \\
\hline Detrimental interactions & 1.0 & 0.6 & & & 1.3 & 0.6 & & & -0.55 & 0.28 & 0.58 & $0.33-0.99$ & 0.05 \\
\hline $\begin{array}{l}\text { Block } 4 \text { Work-related } \\
\text { factors (Nagelkerke's } \\
\mathrm{R}^{2}=0.56 \text { ) }\end{array}$ & & & & & & & & & & & & & $<0.001$ \\
\hline \multicolumn{14}{|l|}{ Employment } \\
\hline Working a & & & 268 & 92.4 & & & 22 & 7.6 & & & & & 0.05 \\
\hline Sick leave & & & 290 & 70.4 & & & 122 & 29.6 & -1.36 & 0.56 & 0.26 & $0.09-0.77$ & 0.02 \\
\hline Unemployed & & & 10 & 20.8 & & & 38 & 79.2 & -1.66 & 1.12 & 0.19 & $0.02-1.68$ & 0.14 \\
\hline $\begin{array}{l}\text { Self-perceived work } \\
\text { ability }\end{array}$ & 3.4 & 1.0 & & & 2.2 & 1.0 & & & 0.41 & 0.22 & 1.50 & $0.99-2.30$ & 0.06 \\
\hline $\begin{array}{l}\text { Intention to return to } \\
\text { work }\end{array}$ & & & 538 & 84.9 & & & 96 & 15.1 & 1.83 & 0.58 & 6.22 & $1.98-19.51$ & 0.002 \\
\hline $\begin{array}{l}\text { Perceived employer } \\
\text { accommodation }\end{array}$ & 3.8 & 0.9 & & & 2.8 & 1.2 & & & 0.66 & 0.16 & 1.93 & $1.41-2.65$ & $<0.001$ \\
\hline Job requirements & 3.8 & 0.9 & & & 2.8 & 1.2 & & & 0.61 & 0.30 & 1.84 & $1.02-3.30$ & 0.04 \\
\hline
\end{tabular}

of physical problems were excluded from the regression model (Nagelkerke's $\left.\mathrm{R}^{2}=0.32\right)(\mathrm{P}<0.001)$. Patients with cancer recurrence or cancer progress had a significant lower chance to return to work compared to patients in cancer remission.

The third block entered consisted of psychosocial factors. The variables fear of recurrence, depression, and social support were excluded from the regression model (Nagelkerke's $\left.\mathrm{R}^{2}=0.39\right)(\mathrm{P}<0.001)$. Individuals with a higher amount of problematic social interactions were less likely to return to work.

The forth block entered consisted of work-related factors. The following variables were excluded from the regression model: duration of sick leave (days) prior to rehabilitation, perceived threat of job loss, expected period of RTW after rehabilitation, and overall job satisfaction. Patients who intended to return to work at baseline were more than six times as likely to do so $(\mathrm{P}<0.001)$. Also, patients who perceived their employer as being cooperative and accommodating of their cancer were more likely to return to work. Although the variable job requirements showed a negative correlation with RTW, it also emerged as a positive predictor for RTW. This is likely an effect of suppression since high job requirements are positively associated with RTW among individuals in the higher social class but negatively associated with RTW among patients in lower social class. When the variable is entered as a 
single factor into a regression analysis, it remains as a negative predictor of RTW $[\beta=-0.41, \mathrm{OR}=0.67,95 \%$ confidence interval $(95 \% \mathrm{CI}) 0.48-0.92, \mathrm{P}=0.01]$. The explained variance of the total model (Nagelkerke's $\mathrm{R}^{2}$ ) was $0.59(\mathrm{P}<0.001)$.

\section{Predictors of time period to RTW}

The following variables remained in the model as significant predictors: UICC cancer stage, Karnofsky index, physical and mental quality of life, employment characteristics, intention to return to work, perceived employer accommodation, and job requirements (overall model fit $-2 \log$ likelihood $=3858.2 ; \chi^{2}[$ total model $]=175.6$, $\mathrm{df}=13, P<0.001)($ table 5).

\section{Discussion}

The majority of patients $(85 \%)$ were motivated either to return to work or be re-employed after rehabilitation. These findings are consistent with results indicating the significance of work and the strong motivation to continue work during treatment or return to work after treatment completion among survivors (41). The exploration of work characteristics revealed that slightly more than half of participants $(55 \%)$ were on sick leave at baseline indicating a high physical and/or psychological symptom burden at the beginning of the rehabilitation program. In our study, the employment rate was $76 \%$ on average 23 months after diagnosis and considerably higher compared to findings showing that overall, about $63.5 \%$ of cancer patients (range 24-94\%) manage to return to work (17). However, comparable results (73\%, range $64-82 \%$ ) have been found at 18 months after the cancer diagnosis among breast cancer patients (14) and among samples with mixed tumor entities (1, $8,25)$. Concordant with previous studies showing work changes in $8-17 \%$ of cancer survivors, we found similar results $(25,30)$.

Both the absence of sick leave and belonging to a higher social class at baseline were associated with a significant higher rate of employment at follow-up. Belonging to a higher social class is related to work environments that may provide more favorable working conditions in terms of flexibility, a lower degree of manual and physically exhausting work, better earnings, and better living conditions. Accordingly, it has been stated that employees with cancer or other persistent health problems generally need some flexibility at various aspects and times at work (42). Atkinson et al (43) and Gudbergson et al (44) pointed out that living conditions include the social indicators that stimulate social inclusion and reduce social exclusion. These indicators are economy, education, employment, health, housing, and social participation.

The highest percentage of patients who did not return

Table 5. Cox's proportional hazards regression model for the identification of significant predictors of the time to return-to-work. [HR=hazard ratio; $\mathrm{SD}=$ standard deviation; $\mathrm{SE}=$ standard error; UICC=International Union against Cancer; $95 \% \mathrm{Cl}=95 \%$ confidence interval.]

\begin{tabular}{|c|c|c|c|c|c|c|c|}
\hline & \multicolumn{2}{|c|}{$\begin{array}{l}\text { Time period (weeks) to } \\
\text { return to work }\end{array}$} & \multirow[t]{2}{*}{$\beta$} & \multirow[t]{2}{*}{ SE } & \multirow[t]{2}{*}{$\mathrm{HR}$} & \multirow[t]{2}{*}{$95 \% \mathrm{Cl}$} & \multirow[t]{2}{*}{ P-value } \\
\hline & Mean & SD & & & & & \\
\hline Months since current diagnosis & & & 0.01 & 0.01 & 1.01 & $0.99-1.03$ & 0.35 \\
\hline \multicolumn{8}{|l|}{ Cancer stage (UICC) a } \\
\hline l b & 13.90 & 19.65 & & & & & 0.02 \\
\hline$\|$ & 18.07 & 21.44 & -0.27 & 0.12 & 0.76 & $0.60-0.97$ & 0.03 \\
\hline III & 20.05 & 22.66 & -0.37 & 0.16 & 0.69 & $0.51-0.94$ & 0.02 \\
\hline IV & 29.18 & 24.74 & -0.59 & 0.26 & 0.56 & $0.34-0.92$ & 0.02 \\
\hline Karnofsky status & & & 0.02 & 0.01 & 1.02 & $1.00-1.04$ & 0.04 \\
\hline Number of functional impairments & & & -0.01 & 0.02 & 0.99 & $0.96-1.02$ & 0.49 \\
\hline Physical quality of life & & & 0.02 & 0.01 & 1.02 & $1.01-1.04$ & 0.001 \\
\hline Mental quality of life & & & 0.01 & 0.01 & 1.01 & $1.00-1.03$ & 0.009 \\
\hline \multicolumn{8}{|l|}{ Employment } \\
\hline Working ${ }^{b}$ & 5.48 & 13.56 & & & & & $<0.001$ \\
\hline Sick leave & 21.61 & 21.37 & -0.99 & 0.16 & 0.37 & $0.27-0.51$ & $<0.001$ \\
\hline Unemployed & 45.67 & 14.45 & -1.41 & 0.41 & 0.25 & $0.11-0.55$ & 0.001 \\
\hline \multicolumn{8}{|l|}{ Intention to return to work } \\
\hline No & 40.53 & 20.18 & -0.49 & 0.20 & 0.61 & $0.41-0.91$ & 0.02 \\
\hline Yes $^{b}$ & 12.59 & 18.38 & & & & & \\
\hline Perceived employer accommodation & & & 0.17 & 0.06 & 1.18 & $1.06-1.32$ & 0.002 \\
\hline Job requirements & & & 0.17 & 0.09 & 1.19 & $1.01-1.41$ & 0.04 \\
\hline
\end{tabular}


to work was observed among patients with lung cancer (43\%) and head and neck cancers (58\%), among patients with advanced cancer stage, progress or metastatic cancer, and palliative treatment intention. The adverse effect of cancer progress and poor physical functioning on RTW has been shown in several previous studies $(7,23$, 27). Corresponding to previous research, we found reemployment significantly associated with younger age and a professional status as an employee $(24,27,45)$.

Our regression analyses findings emphasize the importance of volitional factors for RTW. Patients who expressed their intention to return to work at the beginning of the cancer rehabilitation program were six times more likely to do so compared to patients who did not intend to return to work. Our findings show that the patient can best predict RTW at an early stage of the rehabilitation process. However, so far only limited knowledge exists about motivational and volitional factors and its association with demographic, family and work-related aspects $(46,47)$. Occupational motivation or skepticism towards RTW should be carefully assessed at the beginning of rehabilitation programs and during the joint establishment of rehabilitation aims between the patients and the professional team (48). Furthermore, factors influencing occupational motivation among cancer survivors need to be understood in more detail.

The strong influence of work-related aspects next to medical factors such as disease phase confirm the current state of the literature with regard to factors positively and adversely influencing RTW among cancer survivors $(7,14,23,25,26,45)$. Consistent with the literature, the perceived employer accommodation indicated a fairly positive attitude towards employment, work-related support, and necessary job changes (14, 27,49 ) and emerged as a significant predictor for RTW.

In our study, analyses also demonstrated that the patients who were working at baseline were more likely to return to work after cancer rehabilitation and were more likely to do so at an earlier stage than patients on sick leave or - unsurprisingly - unemployed patients. Self-perceived work ability, in contrast, did not emerge as a significant predictor for RTW. The relevance of perceived work ability has been emphasized in a number of studies $(3,15,44,50-53)$. However, only limited knowledge exists about the association between perceived work ability and sick leave absence.

Furthermore, we found that detrimental social interactions were inversely associated with RTW. Patients with problematic social interactions in their personal environment might also lack social skills at the workplace or might have fewer personal resources to effectively adapt to the challenges of being a cancer survivor in the work environment.

The variable "high job requirements" showed a negative correlation with RTW. However, it also emerged as a positive predictor for RTW likely due to suppression effects. Our findings emphasize that more research is needed particularly to investigate the kind of job requirements (such as having plenty of work or high time pressure) that might lead to early retirement, unemployment, or a higher probability to RTW. Our results point toward the fact that high job requirements are positively associated with RTW among individuals in the higher social class but are negatively associated with RTW among patients in the lower social class. Individuals belonging to a higher social class are more likely to have better working conditions such as flexible work, responsibility, and a considerable amount of decision-making freedom that might compensate the high workload.

Although this prospective study includes a large sample size compared to previous research, this study has several methodological limitations. Both the initial and follow-up non-response lead to a bias in several sociodemographic and psychosocial outcome variables of interest in this research. With regard to the generality and interpretation of the findings, a sample bias must be considered toward: (i) female gender, (ii) younger age, (iii) being in a cancer rehabilitation program, (iv) cancer entities associated with a better physical health status and prognosis, and (v) better psychological well-being. Given this bias, our findings with regard to employment might overestimate the degree to which cancer patients return to work or stay employed. Nevertheless, systematic differences between participants and non-participants were small in view of effect sizes and at least partially a consequence of the relatively large sample size. Although $86 \%$ of the patients were women, the overall large sample size including 107 men justifies gender analyses; however, gender did not correlate with employment status and therefore was excluded from further regression analyses.

Another limitation is that due to the allocation process mainly regulated by the German pension insurance, it was not feasible to randomize the study sample. Despite the fact that cancer rehabilitation programs are provided to every cancer patient in Germany, our sample consists only of patients who use the services provided. This might lead to a bias towards a sample with high physical and psychosocial impairments. Furthermore, the inclusion of a matched control group could not be realized since most patients with rehabilitation needs are referred to a rehabilitation program. Thus, this study could not determine possible effects of the rehabilitation program on RTW.

The (re-)integration of cancer survivors into working life is one important aspect of participation according to the ICF (31). Rehabilitation programs are important not only for the physical and psychosocial recovery, but for the labor market reintegration of patients. Profound understanding of cancer and treatment-induced impairments and their impact on daily activities and work is 
an essential basis for the development of better educational, rehabilitative, and occupational interventions in cancer care (54). A better understanding of cancer and treatment-induced physical, cognitive and psychosocial treatment consequences related to work-related problems will help to develop interventions and educational programs for patients, healthcare professionals, and employers to better address the professional needs of individuals with cancer.

\section{Acknowledgements}

We thank Birgit Leibbrand, MD, Jürgen Barth, MD, Manfred Gaspar, MA, Gerhard Friedrich, MD, Wilhelm Bootsveld, MD, Ulrich Gärtner, MD, and ChristineDaniela Kegel, MD, for their data collection and management support. This research was funded by the Nordrhein-Westfalen Association for the Fight Against Cancer, Germany.

\section{References}

1. Spelten E. Cancer, fatigue and the return of patients to work-a prospective cohort study. Eur J Cancer. 2003;39:1562-7. http://dx.doi.org/10.1016/S0959-8049(03)00364-2.

2. Verbeek J. Return to work of cancer survivors: a prospective cohort study into the quality of rehabilitation by occupational physicians. Occup Environ Med. 2003;60:352-7. http:// dx.doi.org/10.1136/oem.60.5.352.

3. Bradley CJ, Neumark D, Luo Z, Bednarek H, Schenk M. Employment Outcomes of Men Treated for Prostate Cancer. J Natl Cancer I. 2005;97:958-65. http://dx.doi.org/10.1093/ jnci/dji171.

4. Hoffman B. Cancer Survivors at Work: A Generation of Progress. CA-Cancer J Clin. 2005;55:271-80. http://dx.doi. org/10.3322/canjclin.55.5.271.

5. Feuerstein M. Cancer Survivorship and Work. J Occup Rehabil. 2005;15:1-2. http://dx.doi.org/10.1007/s10926005-0868-x.

6. Feuerstein M, Harrington CB. Recommendations for the U.S. National Occupational Research Agenda: Research on Cancer Survivorship and Musculoskeletal Disorders and Work Disability. J Occup Rehabil. 2006;16:1-5. http://dx.doi. org/10.1007/s10926-005-9004-1.

7. Pryce J, Munir F, Haslam C. Cancer survivorship and work: Symptoms, supervisor response, co-worker disclosure and work adjustment. J Occup Rehabil. 2007;17:83-92. http:// dx.doi.org/10.1007/s10926-006-9040-5.

8. Boer AGEM de, Verbeek JHAM, Spelten ER, Uitterhoeve ALJ, Ansink AC, Reijke TM de et al. Work ability and returnto-work in cancer patients. Br J Cancer. 2008;98:1342-7. http://dx.doi.org/10.1038/sj.bjc.6604302.
9. Tiedtke C, Rijk A de, Dierckx Casterlé B de, Christiaens M, Donceel P. Experiences and concerns about 'returning to work' for women breast cancer survivors: a literature review. PsychoOncology. 2010;19:677-83. http://dx.doi.org/10.1002/ pon. 1633 .

10. Feuerstein M, Todd BL, Moskowitz MC, Bruns GL, Stoler MR, Nassif $T$ et al. Work in cancer survivors: a model for practice and research. J Cancer Surviv. 2010;4:415-37. http:// dx.doi.org/10.1007/s11764-010-0154-6.

11. Thijs KM, Boer AGEM, Vreugdenhil G, Wouw AJ, Houterman S, Schep G. Rehabilitation Using High-Intensity Physical Training and Long-Term Return-to-Work in Cancer Survivors. J Occup Rehabil. 2011 Nov 12. [Epub ahead of print]. http:// dx.doi.org/10.1007/s10926-011-9341-1.

12. McGrath PD, Hartigan B, Holewa H, Skarparis M. Returning to work after treatment for haematological cancer: findings from Australia. Support Care Cancer. 2011 Oct 28. [Epub ahead of print] . http://dx.doi.org/10.1007/ s00520-011-1298-2.

13. Roelen CAM, Koopmans PC, Groothoff JW, Klink JJL, Bültmann U. Return to Work After Cancer Diagnosed in 2002, 2005 and 2008. J Occup Rehabil. 2011;21:335-41. http:// dx.doi.org/10.1007/s10926-011-9319-z.

14. Bouknight RR, Bradley CJ, Zhehui L. Correlates of Return to Work for Breast Cancer Survivors. J Clin Oncol. 2006;24:34553. http://dx.doi.org/10.1200/JCO.2004.00.4929.

15. Taskila T, Lindbohm ML. Factors affecting cancer survivors employment and work ability. Acta Oncol. 2007;46:446-51. http://dx.doi.org/10.1080/02841860701355048.

16. Park J, Park E, Park J, Kim S, Lee S. Job loss and re-employment of cancer patients in Korean employees: a nationwide retrospective cohort study. J Clin Oncol. 2008;26:1302-9. http://dx.doi.org/10.1200/JCO.2007.14.2984

17. Mehnert A. Employment and work-related issues in cancer survivors. Crit Rev Oncol Hemat. 2011;77:109-30. http:// dx.doi.org/10.1016/j.critrevonc.2010.01.004.

18. Peteet JR. Cancer and the meaning of work (eng). Gen Hosp Psychiatry. 2000;22:200-5. http://dx.doi.org/10.1016/S01638343(00)00076-1.

19. Rasmussen DM, Elverdam B. The meaning of work and working life after cancer: an interview study. PsychoOncology. 2008;17:1232-8. http://dx.doi.org/10.1002/ pon.1354.

20. Choi KS, Kim E, Lim J, Kim S, Lim MK, Park J et al. Job loss and reemployment after a cancer diagnosis in Koreans - a prospective cohort study. Psycho-Oncology. 2007;16:205-13. http://dx.doi.org/10.1002/pon.1054.

21. Carlsen K, Dalton SO, Diderichsen F, Johansen C. Risk for unemployment of cancer survivors: A Danish cohort study. Eur J Cancer. 2008;44:1866-74. http://dx.doi.org/10.1016/j. ejca.2008.05.020

22. Boer AGEM de, Taskila T, Ojajärvi A, van Dijk FJH, Verbeek JHAM. Cancer survivors and unemployment: a meta-analysis and meta-regression. J Am Med Assoc. 2009;301:753-62. http://dx.doi.org/10.1001/jama.2009.187. 
23. Spelten ER, Sprangers MA, Verbeek JH. Factors reported to influence the return to work of cancer survivors: a literature review. Psycho-Oncology. 2002;11:124-31. http://dx.doi. org/10.1002/pon.585.

24. Taskila-Abrandt T, Martikainen R, Virtanen S, Pukkala E, Hietanen P, Lindbohm M. The impact of education and occupation on the employment status of cancer survivors. Eur J Cancer. 2004;40:2488-93. http://dx.doi.org/10.1016/j. ejca.2004.06.031.

25. Amir Z, Moran T, Walsh L, Iddenden R, Luker K. Return to paid work after cancer: a British experience. J Cancer Surviv. 2007;1:129-36. http://dx.doi.org/10.1007/s11764-0070021-2.

26. Chan F, Strauser D, da Silva Cardoso E, Xi Zheng L, Chan JYC, Feuerstein M. State vocational services and employment in cancer survivors. J Cancer Surviv. 2008;2:169-78. http:// dx.doi.org/10.1007/s11764-008-0057-y.

27. Molina Villaverde R, Feliu Batlle J, Villalba Yllan A, Jimenez Gordo AM, Redondo Sanchez A, San Jose Valiente B et al. Employment in a cohort of breast cancer patients. Occup Med. 2008;58:509-11. http://dx.doi.org/10.1093/occmed/kqn092.

28. Ross L, Petersen MA, Johnsen AT, Lundstroem LH, Carlsen K, Groenvold M. Factors associated with Danish cancer patients' return to work. A report from the population-based study 'The Cancer Patient's World'. Cancer Epidemiol. 2011 Aug 12. [Epub ahead of print].

29. Short PF, Vasey JJ, Tunceli K. Employment pathways in a large cohort of adult cancer survivors. Cancer. 2005;103:1292-301. http://dx.doi.org/10.1002/cncr.20912.

30. Gudbergsson SB, Fosså SD, Dahl AA. A study of work changes due to cancer in tumor-free primary-treated cancer patients. A NOCWO study. Support Care Cancer. 2008;16:1163-71. http://dx.doi.org/10.1007/s00520-008-0407-3.

31. World Health Organization (WHO). ICF-International Classification of Functioning, Disability and Health. Genf; 2001.

32. Hellbom M, Bergelt C, Bergenmar M, Gijsen B, Loge JH, Rautalathi $\mathrm{M}$ et al. Cancer rehabilitation: A Nordic and European perspective. Acta Oncol. 2011;50:179-86. http:// dx.doi.org/10.3109/0284186X.2010.533194.

33. Winkler J, Stolzenberg H. Der Sozialschichtindex im Bundesgesundheitssurvey [The social class index according to the national health survey]. Gesundheitswesen. 1999;61:S178-S183.

34. Karnofsky DA, Burchenal JH. The Clinical Evaluation of Chemotherapeutic Agents in Cancer. In: MacLeod CM, editor. Evaluation of Chemotherapeutic Agents. New York: Columbia University Press; 1949, p. 191-205.

35. Cleeland CS, Ryan KM. Pain assessment: global use of the Brief Pain Inventory (eng). Ann Acad Med Singap. 1994;23:129-38.

36. Herrmann C, Buss U, Snaith RP. HADS-D - Hospital Anxiety and Depression Scale - Deutsche Version: Ein Fragebogen zur Erfassung von Angst und Depressivität in der somatischen Medizin. Bern: Huber; 1995.
37. Mehnert A, Herschbach P, Berg P, Henrich G, Koch U. Fear of progression in breast cancer patients--validation of the short form of the Fear of Progression Questionnaire (FoP-Q-SF). Z Psychosom Med Psychother. 2006;52:274-88.

38. Ware JE, Kosinski M, Dewey JE, Gandek B. How to Score and Interpret Single-Item Health Status Measures: A Manual for Users of the SF-8TM Health Survey. Lincoln, RI: QualityMetric Incorporated; 1999.

39. Ramm GC, Hasenbring M. Die deutsche Adaptation des Illness-specific Social Support Scale und ihre teststatistische Überprüfung beim Einsatz an Patienten vor und nach Knochenmarktransplantation [The German adaptation of the Illness-specific Social Support Scale and the test statistical evaluation on the basis of patients undergoing bone marrow transplantation]. Z Med Psychol 2003;12, 29-38.

40. Bürger W, Dietsche S, Morfeld M, Koch U. Multiperspective estimates on the probability of patient return to work following orthopaedic rehabilitation: findings and predictive relevance. Rehabilitation. 2001;40:217-25.

41. Mahar KK, BrintzenhofeSzoc K, Shields JJ. The impact of changes in employment status on psychosocial well-being: a study of breast cancer survivors. J Psychosoc Oncol. 2008;26:117. http://dx.doi.org/10.1080/07347330802115400.

42. Feuerstein M, Luff GM, Harrington CB, Olsen CH. Pattern of workplace disputes in cancer survivors: a population study of ADA claims. J Cancer Surviv. 2007;1:185-92. http://dx.doi. org/10.1007/s11764-007-0027-9.

43. Atkinson T, Cantillon B, Marlier E, Nolan B. Social indicators The EU and social inclusion. Oxford: Oxford University Press; 2002.

44. Gudbergsson SB, Fosså SD, Borgeraas E, Dahl AA. A comparative study of living conditions in cancer patients who have returned to work after curative treatment. Support Care Cancer. 2006;14:1020-9. http://dx.doi.org/10.1007/s00520006-0042-9.

45. Hensel M. Quality of life and rehabilitation in social and professional life after autologous stem cell transplantation. Ann Oncol. 2002;13:209-17. http://dx.doi.org/10.1093/ annonc/mdf031.

46. Nachreiner N, Dagher R, McGovern P, Baker B, Alexander B, Gerberich S. Successful return to work for cancer survivors. AAOHN J. 2007;55:290-5.

47. Johnsson A, Fornander T, Olsson M, Nystedt M, Johansson H, Rutqvist L. Factors associated with return to work after breast cancer treatment. Acta Oncol. 2007;46:90-6. http://dx.doi. org/10.1080/02841860600857318.

48. Thies S, Lehmann C, Kriz D, Nubling R, Mehnert A. Patientenfragebogen zur Erfassung der Reha-Motivation (PAREMO-20) - Testtheoretische Überprüfung und Validierung an einer Stichprobe von Krebspatienten unterschiedlicher Diagnosegruppen . Rehabilitation. 2008;47:308-18. http:// dx.doi.org/10.1055/s-2008-1076709.

49. Steiner JF, Cavender TA, Nowels CT, Beaty BL, Bradley CJ, Fairclough DL et al. The impact of physical and psychosocial factors on work characteristics after cancer. Psycho-Oncology. 2008;17:138-47. http://dx.doi.org/10.1002/pon.1204. 
50. Short PF, Vasey JJ, BeLue R. Work disability associated with cancer survivorship and other chronic conditions. Psycho-Oncology. 2008;17:91-7. http://dx.doi.org/10.1002/ pon.1194.

51. Lee MK, Lee KM, Bae J, Kim S, Kim Y, Ryu KW et al. Employment status and work-related difficulties in stomach cancer survivors compared with the general population. Br J Cancer. 2008;98:708-15. http://dx.doi.org/10.1038/ sj.bjc. 6604236 .

52. Ahn E, Cho J, Shin DW, Park BW, Ahn SH, Noh D et al. Impact of breast cancer diagnosis and treatment on workrelated life and factors affecting them. Breast Cancer Res Treat. 2009;116:609-16. http://dx.doi.org/10.1007/s10549008-0209-9.
53. Torp S, Nielsen RA, Gudbergsson SB, Dahl AA. Worksite adjustments and work ability among employed cancer survivors. Support Care Cancer. 2011 Nov 16. [Epub ahead of print]. http://dx.doi.org/10.1007/s00520-011-1325-3.

54. Tamminga S, de B, Verbeek J, Taskila T, Frings-Dresen M. Enhancing return-to-work in cancer patients, development of an intervention and design of a randomised controlled trial. BMC Cancer. 2010;10:345. http://dx.doi.org/10.1186/14712407-10-345.

Received for publication: 12 December 2012 